\title{
Trayectorias Laborales de Mujeres y Violencia en el Trabajo: Una Cuestión de Género
}

\section{Women's Work Trajectories and Workplace Violence: A Gender Issue}

\author{
Rosario Undurraga \\ Universidad Finis Terrae \\ Natalia López Hornickel \\ Pontificia Universidad Católica de Chile
}

\begin{abstract}
El reconocimiento de la violencia en el trabajo ha aumentado en los últimos años (Cruz \& Klinger, 2011). Recientemente en Chile, se ha comenzado a visibilizar la violencia de género en el trabajo (Díaz, Mauro, Ansoleaga \& Toro, 2017). En esta línea, este estudio relaciona las trayectorias laborales de mujeres chilenas con la violencia de género en el trabajo. Es un estudio cualitativo que utiliza entrevistas semiestructuradas y biogramas para analizar las trayectorias laborales de mujeres profesionales y no-profesionales, residentes en Santiago de Chile. La muestra por conveniencia consta de 7 mujeres (24-62 años) que reportaron eventos de violencia laboral como hitos fundamentales en sus trayectorias. Se identificaron 3 tipos de violencia de género en el trabajo: (a) trato condescendiente, (b) discriminación frente a la maternidad y (c) acoso sexual. Ante estos hechos, se encuentran reacciones de resignación y resistencia, variando según el tipo de violencia, nivel educacional y experiencias subjetivas de las mujeres. El acoso sexual produce cambios laborales, siendo un punto de inflexión en sus trayectorias laborales. Se concluye que la violencia en el trabajo es típicamente una cuestión de género. Se insta a generar una cultura laboral libre de violencia de género.
\end{abstract}

Palabras clave: violencia laboral, discriminación, acoso, Chile, mujer

\begin{abstract}
The recognition of workplace violence has increased in recent years (Cruz \& Klinger, 2011). In Chile, gender-based violence at work has become more visible (Díaz, Mauro, Ansoleaga \& Toro, 2017). In this context, this article examines the relationship between Chilean women's work trajectories and gender violence at work. This is a qualitative study that employs semi-structured interviews and biograms to analyze the work trajectories of professional and non-professional women residing in Santiago de Chile. The convenience sample used comprises 7 women (24-62 years old) who reported incidents of workplace violence as milestones in their work trajectories. Three types of gender-based workplace violence were identified: (a) condescending treatment, (b) discrimination due to maternity, and (c) sexual harassment. In response to these events, participants report reactions of resignation and resistance, which varied according to the type of violence involved, the workers' educational level, and their subjective experiences. Sexual harassment produces work changes, representing a turning point in women's work trajectories. It is concluded that workplace violence is generally a gender issue, which stresses the importance of generating a work culture free from gender-based violence.
\end{abstract}

Keywords: workplace violence, discrimination, harassment, Chile, women

La violencia en el trabajo ha sido definida por la Organización Internacional del Trabajo (OIT) como "toda acción, incidente o comportamiento que se aparta de lo razonable mediante el cual una persona es agredida, amenazada, humillada o lesionada por otra en el ejercicio de su actividad profesional o como consecuencia directa de la misma" (OIT, 2003, p. 4).

La violencia en el trabajo tiene un carácter multideterminado, transversal y multicausal (Toro \& Gómez-Rubio, 2016) y tiene implicancias que suelen ser soslayadas. En términos laborales, disminuye la productividad, afecta la competitividad y el desarrollo laboral (Cruz \& Klinger, 2011). A nivel organizacional, genera aumento de licencias médicas y rotación laboral, así como también merma la eficacia

Rosario Undurraga, Escuela de Ciencias de la Familia, Universidad Finis Terrae, Santiago, Chile; Natalia López Hornickel, Facultad de Educación, Pontificia Universidad Católica de Chile, Santiago, Chile.

Las autoras agradecen el apoyo del Gobierno de Chile para financiar esta investigación, FONDECYT 11150862.

La correspondencia relativa a este artículo debe ser dirigida a Rosario Undurraga, Escuela de Ciencias de la Familia, Facultad de Educación, Psicología y Familia, Universidad Finis Terrae, Avda. Pedro de Valdivia 1509, Providencia, Santiago, Chile. E-mail: mrundurraga@uft.cl 
laboral (Ansoleaga, Gómez-Rubio \& Mauro, 2015; Palma, Ansoleaga \& Ahumada, 2018). A nivel personal, influye en la salud mental y física (Ansoleaga et al., 2015; Palma et al., 2018; Parra Osorio \& Acosta Fernández, 2010). La violencia afecta la percepción de agencia y autoeficacia (Macmillan, 2001), deteriora la seguridad, confianza, dignidad y autonomía de las víctimas, disminuye las oportunidades laborales, dadas las consecuencias mentales y físicas que conlleva (Artigas, 2007; Cruz \& Klinger, 2011), y puede estar vinculada a cambios en las vidas de los trabajadores, en las expectativas interpersonales y en la estructura de identidad (Goldblatt \& Buchbinder, 2003).

A nivel internacional, la violencia en el trabajo se ha considerado como una responsabilidad del espacio laboral; este sería un lugar donde es factible sufrir discriminación y ser estigmatizado (Summers et al., 2018), sobre todo en el caso de las mujeres y minorías de distinto tipo (Triana, Jayasinghe \& Pieper, 2015). Las políticas organizacionales tendrían un efecto importante en la prevención de estos comportamientos y en la motivación de los empleados, lo que afecta su desempeño laboral (Chang, Eatough, Spector \& Kessler, 2012). A su vez, se ha analizado cómo los efectos de las agresiones dependen de quien las lleve a cabo, siendo las más perjudiciales aquellas perpetradas por los supervisores (Hershcovis \& Barling, 2010).

La literatura sobre violencia laboral en América Latina es reciente (Ansoleaga et al., 2015; Parra \& Acosta, 2010). En Chile, últimamente se ha comenzado a reconocer la violencia laboral como un problema relevante a nivel nacional. Existen publicaciones sobre violencia en el trabajo, particularmente en el área de la salud (Ansoleaga et al., 2015; Ansoleaga \& Urra, 2015; Palma et al., 2018; Parra \& Acosta, 2010; Poblete Troncoso \& Valenzuela Suazo, 2005) y en relación a las emociones (Vandevelde-Rougale \& Guerrero Morales, 2019). Entre hallazgos relevantes, se ha observado cómo la intersección entre la asimetría de poder en el trabajo y la desigualdad de género se traduce en distintos tipos de violencia laboral (Díaz et al., 2017).

En este marco sobre violencia laboral, uno de los tipos de violencia que ha concitado mayor atención es la violencia contra la mujer, la que puede ser comprendida como violencia física, sexual o psicológica y/o amenazas de violencia física o sexual, acoso y abuso psicológico y emocional (Kilpatrick, 2004; Krug, Dahlberg, Mercy, Zwi \& Lozano, 2003; Organización Mundial de la Salud, 2018). La violencia de género refleja y refuerza desigualdades entre hombres y mujeres (Cruz \& Klinger, 2011), reafirmando distintas posiciones de poder. Hace referencia a las definiciones y relaciones de género dominantes en una sociedad determinada, lo que destaca el fundamento sociocultural de este tipo de violencia (Espinar Ruiz \& Mateo Pérez, 2007).

A nivel internacional, se han pactado directrices para eliminar la violencia contra las mujeres, incluyendo mecanismos de protección y seguimiento. La Convención Interamericana para Prevenir, Sancionar y Erradicar la Violencia contra la Mujeres (Convención do Belém do Pará firmada en 1994) establece el derecho de las mujeres a vivir una vida libre de violencia y considera la violencia como una violación de los derechos humanos y de las libertades fundamentales (Organización de los Estados Americanos, 2018). Chile ha firmado y ratificado dicha Convención. Asimismo, en el año 2015, la Organización de las Naciones Unidas (ONU) aprobó la Agenda 2030 sobre el Desarrollo Sostenible. Entre los objetivos de desarrollo sostenible, el objetivo 5 "lograr la igualdad entre los géneros y empoderar a todas las mujeres y las niñas" sostiene que la igualdad de género es la base para una sociedad pacífica, próspera y sostenible (ONU, 2018).

Estas regulaciones se forjan en el contexto social en que existe una dominación masculina o patriarcado, que es un sistema sexo-género basado en prácticas y estructuras sociales jerárquicas caracterizadas por relaciones de poder, donde el hombre domina y tiene autoridad por sobre la mujer (Cockburn, 1983; Walby, 1990). Así, la violencia de género considera todas las formas de violencia que se encuentran enraizadas en alguna forma de ideología patriarcal, que puede ser llevada a cabo por mujeres y hombres, contra mujeres y/o hombres, con el propósito de mantener el poder social de los hombres (Cruz \& Klinger, 2011). Las estructuras patriarcales son formas de violencia basadas en el género (Espinar Ruiz \& Mateo Pérez, 2007).

Actualmente, la violencia de género es descrita como la violación a los derechos humanos más prevalente en el mundo: al menos una de tres mujeres es víctima de un abuso sexual, físico o de otro tipo a lo largo de su vida (Cruz \& Klinger, 2011). Su predominio no ha dejado incólume al ámbito laboral. Las mujeres se ven expuestas a abusos de tipo físico, psicológico o sexual en el trabajo, dada su desigual posición en la sociedad (Chappell \& Di Martino, 2006; International Labour Organization [ILO], 2016). En Chile, Díaz et al. (2017) señalan que la violencia de género en el trabajo es propiciada en el marco de relaciones laborales con un pronunciado desbalance de poder entre ejecutivos y trabajadores, con estilos de 
liderazgo y supervisión autoritarios, donde las mujeres tienden a ingresar a ocupaciones con menor salario y enfrentar mayores dificultades para acceder a posiciones de poder. Esto implica que la violencia de género en el trabajo emerge en determinados contextos organizacionales que lo (re)producen (Artigas, 2007; Calvin Pérez et al., 2013).

En el presente estudio se definió violencia de género en el trabajo como toda acción o conducta que implique abuso, desmedro, menoscabo y/o perjuicio en contra de una persona, en base al género, y que ocurre en referencia al ámbito laboral. Adscribe al término violencia de género, ya que los abusos contra las mujeres se relacionan directamente con desigualdades de poder entre los géneros (Díaz et al., 2017); dichas diferencias están asentadas en lo que se espera que hagan y sean mujeres y hombres en un contexto y sociedad determinados. Es violencia de género en el trabajo, pues esto ocurre en relación a lo laboral. Así, en las condiciones actuales, las mujeres tienen mayor riesgo de experimentar situaciones de violencia que sus pares hombres (Ansoleaga Moreno, Díaz Berr \& Mauro Cardarelli, 2016) y una mayor probabilidad de sufrir discriminación en el espacio laboral (Triana et al., 2015; Undurraga, 2018, 2019a). Díaz et al. (2017) concluyen que "las mujeres están doblemente expuestas a violencia laboral por un bajo poder formal en la estructura ocupacional y por un bajo poder social que emana de relaciones de género que subordinan y discriminan a las mujeres" (p. 44). A su vez, en el marco de labores que involucran interacción con hombres violentos, las mujeres pueden verse afectadas en mayor medida que los hombres (Seymour, 2009), impactando en sus patrones de empleo y en su salud (Lloyd \& Taluc, 1999).

La violencia de género en el trabajo se relaciona con la división sexual tradicional del trabajo, que asigna a los hombres el ámbito de la producción y a las mujeres, el de la reproducción; el espacio laboral tensiona estos roles tradicionales de género, dado que incorpora a las mujeres al ámbito de la producción (Riquelme Giagnoni, 2011). El mercado laboral es construido desde una lógica masculina, presentando resistencias a la participación femenina. Además, el modelo masculino de trabajo genera una imagen de trabajador ideal que supone disponibilidad para trabajar a tiempo completo y de manera continua, sin responsabilidades de cuidado (Acker, 1990; Lewis, 1997). Estas condiciones diferenciadas en base al género podrían considerarse un hecho de violencia en sí mismo, pues afectan el ingreso, desarrollo y salida del mercado laboral en base al género, siendo las mujeres perjudicadas consistentemente en distintas etapas de su trayectoria. En efecto, en Chile, las mujeres sufren severa discriminación para ingresar a trabajar (Undurraga, 2018, 2019a) y para ascender a puestos de mayor jerarquía (Undurraga \& Barozet, 2015), mientras los hombres se encuentran sobrerrepresentados en posiciones de poder (Cárdenas Tomažič, 2018; Virtus Partners \& ComunidadMujer, 2018).

Respecto de las organizaciones, estas no son neutrales en términos de género (Acker, 2006). West y Zimmerman (1987) argumentan que el género está presente en las interacciones cotidianas; emerge de las instituciones sociales y de la interacción social, de manera tal que se mantienen, (re)producen y legitiman formas de organizar la vida social. Por tanto, en el ámbito del trabajo también se (re)produce el sistema de relaciones sociales y de género, por medio de las instituciones que lo sustentan.

Este estudio abordó la pregunta: ¿cómo incide la violencia de género en el trabajo en la trayectoria laboral de mujeres chilenas? Tuvo por objetivo indagar en la violencia de género en el trabajo y sus distintos tipos de manifestaciones y relacionar estas experiencias de violencia con las decisiones de las mujeres afectadas, observando los efectos en sus trayectorias laborales.

La relevancia social de estudiar la violencia laboral y, en particular, la violencia de género en el trabajo, radica en la tendencia generalizada al alza de la violencia y las graves consecuencias que tiene a nivel personal y organizacional (Chang et al., 2012; Palma et al., 2018). En un país con una baja tasa de participación laboral femenina - 49,3\% mujeres versus 69,3\% hombres- (Chile, Instituto Nacional de Estadísticas [INE], 2019) y donde la posición de la mujer se ve entredicha en el mercado laboral (ComunidadMujer, 2018; Programa de las Naciones Unidas para el Desarrollo, 2010; Undurraga, 2013), es relevante visibilizar este problema para generar mayor concientización de estas prácticas y tomar medidas preventivas para promover una participación laboral libre de violencia.

\section{Trayectoria Laboral}

En esta investigación se utiliza el concepto de trayectoria para estudiar el efecto de la violencia en el trabajo en la vida laboral de mujeres. La trayectoria se refiere a una secuencia de eventos de vida, transiciones y etapas de vida, donde las transiciones son cambios de un estado o período a otro y las etapas 
son fases relativamente estables entre dos transiciones (Levy \& Pavie Team, 2005). La complejidad contenida en el fenómeno de la violencia se intersecta con aquella que caracteriza la trayectoria laboral de una persona. Cada trayectoria se (re)construye a partir de los significados que las personas otorgan a sus experiencias, en cada momento de su ciclo vital. Esto se da en diferentes condiciones y oportunidades de trabajo que el mercado laboral demanda y ofrece (Guzmán \& Mauro, 2001). Para aproximarse a las trayectorias laborales de mujeres en Chile, en este estudio se consideraron los momentos más importantes de la vida laboral que las participantes reportaron, tales como trabajos, inactividad, estudios y capacitaciones.

El estudio de las trayectorias laborales ayuda a comprender el mundo del trabajo y sus dinámicas, pues aborda las relaciones entre cambios socioeconómicos y culturales, así como las transformaciones de las subjetividades personales y sociales (Guzmán \& Mauro, 2001). Las representaciones sociales y los significados que los sujetos y colectividades le atribuyen al trabajo son centrales en la configuración de dichas trayectorias (Mauro, 2004).

La trayectoria laboral estaría caracterizada por "un modo estandarizado en que las personas deben organizar diacrónica, secuencial y biográficamente sus acciones en el mercado laboral" (Cárdenas, Undurraga, Altschwager, Leiva, Badilla \& Prado, 2014, p. 14). Es decir, la participación laboral, las razones para ingresar (o no) al mercado laboral, su desarrollo, permanencia, movilidad y la finalización de la misma, se encontrarían socialmente delimitadas, siendo la educación formal un paso previo para el despliegue de la trayectoria laboral (Cárdenas et al., 2014). Sin embargo, existen tensiones y desajustes entre la preparación formal de los jóvenes y el acceso a empleos de calidad (Sepúlveda, 2016). En el caso de las mujeres, las tensiones entre distintas esferas suelen cruzar toda la trayectoria laboral y vital (Todaro \& Yáñez, 2004; Undurraga, 2013).

La investigación en torno a las trayectorias laborales en Chile incluye estudios centrados en la comparación intergeneracional de mujeres (ComunidadMujer, 2018; Guzmán, Mauro \& Araujo, 1999; Guzmán \& Mauro, 2001, 2004; Madero-Cabib, Undurraga \& Valenzuela, 2019), el análisis de trayectorias laborales juveniles (Castillo \& Cabezas, 2010; De la Lastra \& Campusano, 2006; Sepúlveda, 2016), el análisis de trayectorias laborales de la clase media-baja en Chile (Cárdenas et al., 2014) y estudios de trayectorias laborales vinculadas a la previsión social (Mauro \& Yáñez, 2005; Undurraga \& Becker, 2019; Undurraga, 2019b). No obstante, no hay evidencia sustantiva que vincule las trayectorias laborales con la violencia de género en el trabajo.

Para aproximarse a las trayectorias laborales de mujeres en Chile y los efectos de la violencia de género en el trabajo, se consideraron los eventos que las propias participantes destacaron como relevantes en su vida laboral, tanto en la inserción, permanencia y movilidad, como en la salida del mercado laboral. Se consideraron componentes que permitieran reconocer las características, así como continuidades y cambios de cada trayectoria, a saber, número y tipo de empleos, eventos laborales de inserción y retiro, permanencia y transiciones, entre otros. Asimismo, se identificaron decisiones y posibles estrategias en el marco de la experiencia subjetiva de violencia, reconociendo diversas formas de enfrentar la violencia de género en el trabajo.

\section{Diseño}

\section{Método}

Esta investigación cualitativa aborda la relación entre violencia de género en el trabajo y trayectoria laboral de mujeres chilenas. Epistemológicamente, pretende visibilizar la voz de mujeres dando a conocer sus experiencias y miradas respecto del trabajo (Undurraga, 2012).

\section{Participantes}

La muestra contempló la participación de 50 mujeres residentes en Santiago de Chile: 25 profesionales y 25 no-profesionales, con y sin trabajo remunerado, entre 24 y 88 años de edad, distribuidas igualmente en cinco grupos etarios: 20-35, 36-45, 46-60, 61-75 y 76 años y más. Cada grupo estaba compuesto por cinco profesionales y cinco no-profesionales. Se realizó un muestreo intencional, siguiendo criterios de conveniencia, según los criterios de inclusión antes descritos para cada grupo. Se utilizaron informantes clave, contactos del equipo investigador y la estrategia bola de nieve para acceder a las participantes, quienes fueron contactadas por los/as investigadores/as para acordar una entrevista. 
Del total de 50 mujeres, siete participantes mencionaron explícitamente hechos de violencia como eventos fundantes en su trayectoria laboral; estos casos son los considerados en el análisis. De las siete participantes que reportaron violencia laboral, tres son profesionales y se desempeñaban como ingeniera industrial, profesora y licenciada en historia, mientras cuatro de ellas no son profesionales y trabajaban como analista contable, cajera recepcionista y secretaria. Al momento de las entrevistas, seis de ellas trabajaban de manera dependiente y una estaba cesante (ver Tabla 1).

Tabla 1

Caracterización de la Muestra

\begin{tabular}{rccccccc}
\hline ID & Edad & $\begin{array}{c}\text { Nivel } \\
\text { educacional }\end{array}$ & Ocupación & Estado civil & $\begin{array}{c}\mathrm{N}^{\circ} \\
\text { hijos/as }\end{array}$ & $\begin{array}{c}\text { Edad } \\
\text { hijos/as }\end{array}$ & $\begin{array}{c}\text { Situación } \\
\text { ocupacional }\end{array}$ \\
\hline 4 & 29 & $\mathrm{P}$ & Historiadora & Conviviente & 0 & -- & $\begin{array}{c}\text { No trabaja } \\
\text { actualmente }\end{array}$ \\
6 & 29 & $\mathrm{NP}$ & Secretaria & Conviviente & 0 & -- & Dependiente \\
7 & 24 & $\mathrm{NP}$ & Analista contable & Conviviente & 1 & 4 & Dependiente \\
8 & 30 & $\mathrm{NP}$ & Cajera recepcionista & Casada & 1 & 2 & Dependiente \\
21 & 54 & $\mathrm{P}$ & Ingeniera civil industrial & Casada & 0 & -- & Dependiente \\
25 & 50 & $\mathrm{P}$ & Profesora & Separada & 2 & 27,19 & Dependiente \\
36 & 62 & $\mathrm{NP}$ & Secretaria & Casada & 2 & 26,25 & Dependiente \\
\hline
\end{tabular}

Nota. ID: identificación; P: profesional, NP: no profesional.

\section{Instrumento}

Para reconstruir las trayectorias laborales de las participantes, se utilizaron biogramas y entrevistas semiestructuradas. El biograma es una representación gráfica que la participante realiza de su historia laboral (Undurraga \& Calvo Miranda, 2019, Abril). La entrevista se basó en una pauta con dimensiones a indagar sobre la trayectoria laboral. La conversación se estructuró en función de los momentos laborales que las participantes definieron como relevantes a lo largo de su vida laboral, retomando el biograma para aludir a dichos eventos destacados por ellas. Cuando una participante mencionó alguna situación de violencia como hito relevante en su trayectoria laboral, se indagó sobre esa experiencia y su percepción ante ese hecho.

\section{Procedimiento}

Las entrevistas fueron llevadas a cabo por el equipo investigador de manera presencial e individual una a una: un/a entrevistador/a, una participante. Se realizaron entre abril de 2016 y mayo de 2017, en el lugar más conveniente para cada participante (su casa, un café, su trabajo) y duraron entre 46 minutos y 1 hora 44 minutos, en promedio 1 hora 11 minutos. Las entrevistas fueron grabadas y transcritas textualmente.

Todas las entrevistas se realizaron previa firma de un consentimiento informado que asegura el resguardo de la identidad de las participantes. Se les informó sobre los objetivos del estudio, garantizándoles las condiciones de voluntariedad, anonimato y confidencialidad en el manejo de los datos. Esta investigación contó con la aprobación del Comité de Ética de la Universidad Finis Terrae y del Fondo Nacional de Desarrollo Científico y Tecnológico de la Comisión Nacional de Investigación Científica y Tecnológica.

\section{Estrategia de Análisis}

El material recogido en las entrevistas se analizó en base a la teoría fundada (grounded theory; Glaser \& Holton, 2004; Glaser \& Strauss, 1967). Esta visión metodológica permite tanto una aproximación flexible 
al material empírico como un diálogo y contraste entre empiria y teoría. Esto implica que las categorías de análisis emergen a medida que se analiza la información (Krause, 1995), lo que puede relevar aristas no previstas y destacar la visión de las mujeres en su recuento y valoración de sus trayectorias laborales. Se transcribieron todas las entrevistas de forma textual (verbatim). Para el proceso de análisis y codificación se utilizó el software Atlas.ti (ATLAS.ti Scientific Software Development GmbH, 2020) como herramienta de apoyo para levantar códigos, categorías y graficar relaciones entre las respuestas.

Una vez que se remarcaron citas y se levantaron códigos, se desarrolló un ejercicio comparativo que permitió cotejar los datos codificados con los siguientes. Así, las ideas generadas a partir de los primeros inputs de información fueron complementadas con los hallazgos siguientes. Esta metodología implica un proceso iterativo, que da espacio para alcanzar un nivel de saturación teórica, instancia en que los datos no agregan información nueva (Glaser \& Holton, 2004; Krause, 1995). Así, durante el análisis, y con el objetivo de comprender con mayor profundidad la información, mediante el Atlas.ti se desarrollaron mapas gráficos con las categorías que emergieron para tener una perspectiva más amplia de los resultados que surgieron.

\section{Resultados}

Las experiencias de violencia relatadas por las participantes emergieron como hitos relevantes en las trayectorias laborales de siete mujeres, profesionales y no-profesionales y de distinto grupo etario, quienes destacaron estos hechos como aspectos cruciales en su vida laboral. Lo expresado por las participantes y el impacto de estos eventos de violencia en las vidas de estas personas es tal que conllevó implicancias significativas en sus trayectorias laborales. Si bien siete casos es una muestra pequeña, por lo cual los resultados a continuación deben ser considerados con cautela, es importante recordar que el objetivo de una investigación cualitativa no es alcanzar representatividad ni generalización de sus resultados, sino abordar en profundidad un fenómeno social en particular (Flick, 2004; Hernández Sampieri, Fernández Collado \& Baptista Lucio, 2014). Con todo, lo que ocurre en un grupo específico, puede ser iluminador para comprender dinámicas que suceden en otros grupos con características similares.

A partir de los relatos de las participantes, se identificaron tres tipos de manifestaciones de violencia de género en el trabajo: (a) trato condescendiente en la realización de su labor, (b) discriminación frente a la maternidad y (c) acoso sexual. Estos resultados son coherentes con otros estudios sobre violencia de género en el trabajo en Chile (Díaz et al., 2017). Además, fue posible indagar los efectos de estos tipos de violencia en las decisiones laborales de las afectadas. A continuación, se aborda cada uno de estos tipos de violencia y su relación con la trayectoria laboral de estas mujeres.

Las participantes están asociadas a un número de identificación (ID), señalando en las citas su edad, profesión/oficio/trabajo, situación de pareja y número de hijos.

\section{Trato Condescendiente}

El trato condescendiente contempla relaciones laborales hostiles, en las que las mujeres son vistas como menos capaces y se las limita a tareas de servicio que no tienen relación con sus funciones. Son juzgadas, excluidas de la toma de decisiones y postergadas en pos de colegas hombres. Por ejemplo, una ingeniera industrial relata el trato que recibían por parte de colegas hombres, haciéndolas ver como menos capaces:

... nos rayaban las cosas (...) ellos decían "pero ya, escríbeme esto" (...) era bien déspota el trato (...) no había la relación de decir, pucha, no les voy a rayar la hoja tanto, pa' que no se sientan tan tontas. (ID21, 54 años, ingeniera civil industrial, casada, sin hijos)

El género en intersección con la edad puede generar una visión paternalista que infantiliza y menosprecia la capacidad intelectual de las mujeres. La cita a continuación de una profesional historiadora muestra este trato condescendiente:

Los hombres (...) tienen un problema de machismo gigante y te ven como niña chica (...) sienten que tu capacidad intelectual no es la misma que ellos tienen y no es la misma que va a tener un varón de tu misma edad (...) y no solo por la edad, es una serie de creencias (...) no te dan ese espacio para dialogar, porque te desacreditan: "erís [eres] mina [mujer], erís loca, como que andai con la regla" (...) ellos efectivamente sentían que yo servía para ciertas pegas [trabajos] que estaban más relacionadas con ser una secretaria (...) que hacer pegas de investigación y, por ejemplo, hablando de preparación académica, yo tenía mucha más que mi compañero de trabajo. (ID4, 29 años, historiadora, soltera, sin hijos) 
Otras participantes manifiestan haber sido calladas, limitando su desarrollo laboral y confinándolas a tareas de servicio, como lo comenta una analista contable:

Mi jefe era un gallo [hombre] raro, entonces no dejaba que yo hablara, que yo me riera, que diera opiniones. Lo único que cuando necesitaba un favor, como que "ah, la [nombre de ID7] lo puede hacer" (...) él le daba más la preferencia a mi otro compañero que era hombre (...) a mí no me dejaba opinar nada, siendo que llevaba, no sé, poh, un año de trabajo y mi compañero había llegado recién y no sabía el método de trabajo. (ID7, 24 años, analista contable, conviviente, 1 hijo)

En cuanto al efecto en las trayectorias laborales de estas afectadas (ID4 e ID7), estos eventos los manifestaron como experiencias en que se sintieron constantemente desacreditadas y menospreciadas en su trabajo, aunque no se tradujeron en una decisión de salida, pese a que, en el caso de las más jóvenes, sus trayectorias son dinámicas y transitan con frecuencia de un trabajo a otro. Por ejemplo, en estos casos, a su corta edad (29 y 24 años, respectivamente) ya habían pasado por al menos cinco trabajos y no buscaban necesariamente estabilidad laboral.

El trato condescendiente también involucra situaciones amenazantes y tratos inadecuados vinculados a ambientes de trabajo en contextos vulnerables, donde ser mujer con una postura disidente, respecto a la de sus jefes, constituye un factor de riesgo. Por ejemplo, una profesora describe un gesto violento y sorpresivo recibido por parte del director del colegio en el que trabajaba:

Estábamos en el comedor almorzando, tomando desayuno, no sé, y de repente él, para hacerse el gracioso, a raíz de nada, de la cocina pesca un cuchillo así [gesto con la mano] y me lo pone en el cuello. (ID25, 50 años, profesora, separada, 2 hijos)

El trato condescendiente se relaciona con el machismo. Por el hecho de ser mujer, pares o jefes varones las tratan de forma violenta: menosprecio, amenazas, abuso de poder, paternalismo, entre otras. Ante experiencias de este tipo, varias participantes señalan tomar acciones. Algunas cuentan que se defienden, como lo relata esta ingeniera industrial:

Yo tengo un carácter fuerte, entonces, conmigo era diferente, yo no les aguantaba nada (...) siempre me enfrenté a ellos, yo nunca dejé que nadie me pusiera la pata encima (...) les fui demostrando mis capacidades y así yo fui escalando. (ID21, 54 años, ingeniera civil industrial, casada, sin hijos)

Otras participantes intentan gestionar soluciones, como la profesora amenazada por el director, quien conversó con su jefe de ciclo:

Cuando yo hablé con este, "ya" me dijo y después de ahí empezaron a hacer gestiones para sacarlo, como que ahí creyeron. Me imagino que no fui la única que habló. (ID25, 50 años, profesora, separada, 2 hijos)

Este tipo de reacciones fue más común entre las profesionales - al menos dos de tres profesionales de la muestra señalaron sufrir este tipo de violencia - que entre no-profesionales. Estas últimas, a pesar de considerar la situación como non-grata y aplastante, se resignaron. Por ejemplo, el caso descrito de la analista contable (ID7), no refiere a cambios de trayectoria producto del trato condescendiente por parte de su jefe ni tampoco comenta estrategias o soluciones ante el hecho de ser callada y relegada por la autoridad. Más bien, atribuye su salida a una causa externa - al cambio de jefatura-, por lo que prevalece una actitud de resignación respecto de las condiciones en las que desarrolla su trabajo.

En síntesis, estas experiencias de relegación, menosprecio y trato inadecuado por el hecho de ser mujer que han manifestado estas participantes, han interferido en sus trayectorias laborales. Dichas situaciones muestran una alteración de las condiciones mínimas de seguridad e integridad que un espacio laboral debiese otorgar a sus trabajadores.

\section{Discriminación Frente a la Maternidad}

La discriminación frente a la maternidad o mobbing maternal se refiere a la violencia o acoso psicológico laboral que sufre la mujer por el hecho de ser madre (Riquelme Giagnoni, 2011). Esta puede incluir la limitación de funciones, reducción de interacciones laborales, hostigamiento, discriminación por utilizar la hora legal de amamantamiento, entre otros. Se trataría de un acoso psíquico para evitar nuevos embarazos en la organización (Piñuel, 2012). Así lo han vivido participantes de este estudio:

Yo creo que a todas las mujeres embarazadas como que las molestan (...) hubo como un período cuando yo estuve embarazada (...) que tenía la jefa y como que te hinchaba [fastidiaba], te hinchaba, te hinchaba todo el rato. (...) Antes no. (...) Había compañeras que igual estaban embarazadas y sí tiraban [presentaban] licencias 
UNDURRAGA Y LÓPEZ HORNICKEL

[médicas]. Yo creo que a veces igual como que se sobrepasan y todo, pero no por eso te van a empezar a molestar y acosar en ese sentido. (ID8, 30 años, cajera recepcionista en institución de salud, casada, 1 hijo)

... y el 22 de enero de ese mismo año yo supe que estaba embarazada. Entonces como que ahí cortaron todas las relaciones, o sea, yo iba como a cumplir horario (...) era como que me hicieron la ley del hielo (ID7, 24 años, analista contable, conviviente, 1 hijo)

En cuanto a la relación entre la discriminación frente a la maternidad y las trayectorias laborales de las mujeres afectadas, algunas participantes señalan no haber reivindicado sus derechos por falta de opciones laborales y la necesidad de tener un trabajo, limitando sus posibilidades de agencia. Por ejemplo, una participante que trabajaba como secretaria en un medio de prensa escrita, al tener hijos, relata las dificultades que vivió para desempeñar su trabajo y que, por miedo y por conservar su trabajo, nunca ejerció su derecho:

... era mal visto tomarse la hora de amamantamiento (...) nunca me tomé esa hora. (ID36, 62 años, secretaria, casada, 2 hijos)

Asimismo, otras participantes, ante la posibilidad de ser maltratadas por hacer uso de licencia médica, intentan solucionar "temas del embarazo" fuera del horario laboral:

En realidad, me habré tomado durante el embarazo dos permisos, una cosa así (ID7, 24 años, analista contable, conviviente, 1 hijo)

Estas participantes que sufrieron discriminación frente a la maternidad tienen un nivel educacional similar (no-profesionales) y su ocupación se relaciona con servicios de gestión y administración. Respecto al impacto del mobbing maternal en la trayectoria laboral de estas mujeres, las experiencias de discriminación y presiones no concluyeron directamente en la salida laboral. Lo más frecuente fue la resignación, incluso en mujeres de distintas generaciones (grupo 20-35 años y 61-75 años). Esto da luces de que la subordinación de género sería transversal a la edad: la posición de las mujeres en la sociedad chilena y en el espacio laboral reduciría las capacidades de agencia para hacer frente a estas situaciones de discriminación. Este aspecto unifica a mujeres con diferentes particularidades.

\section{Acoso Sexual}

El acoso sexual en el trabajo también forma parte de las experiencias relatadas por algunas entrevistadas. Este se define como aquellos requerimientos indebidos de carácter sexual no consentidos por la persona afectada, que amenacen o perjudiquen su situación laboral o sus oportunidades en el empleo (Dirección del Trabajo, 2019). Esta conducta crea un entorno laboral intimidatorio, hostil y humillante para la persona que es objeto del acoso. Ante experiencias de este tipo, las afectadas se sienten vulneradas y ven reducidas sus posibilidades de desarrollo laboral. Son desacreditadas y finalmente deciden retirarse de su lugar de trabajo. Este tipo de violencia en el trabajo incide drásticamente en la trayectoria laboral de estas participantes, dejando su fuente laboral. Por ejemplo, una cajera recepcionista relata haber sufrido acoso sexual, experiencia frente a la cual no recibió ayuda y decidió retirarse de ese trabajo:

Seis meses estuve ahí, más o menos, con boleta. (...) Por lo mismo, porque me acosaba un compañero (...) un día me agarró la pierna cuando nos íbamos para allá para el [institución pública] y me bajé del auto y me vine en micro. (...) Y hablé con mi jefa, pero no hizo nada. No creyó ni nada, así que ahí después decidí ya salirme de ese trabajo. (...) Me sentía agobiada, me sentía mal. Desesperada igual a veces, porque no sabes cómo reaccionar a algo así; tú pensai en... no sé, en gritar, en decir "¿qué es lo que te está pasando?", pero te cohíbes y no puedes hacer nada. (...) Después, ya no seguí trabajando en eso, en lo mismo. Ya me salí de eso y no quise trabajar más en oficina por miedo a que me pasara lo mismo. (...) No, por eso no trabajé más en oficina. [Mejor] en un recinto abierto donde hubiera más gente. (ID8, 30 años, cajera recepcionista, casada, 1 hijo)

En el caso de la participante ID8, si bien intentó buscar apoyo y seguridad en su jefatura, el retiro laboral fue inminente, dada la ausencia de respuesta de sus superiores y la falta de protección ante un hecho de violencia. Esto da cuenta de la relevancia de las condiciones laborales, así como de la necesidad de regulaciones en estas materias. Además, en este caso, ella dejó de trabajar en oficina o lugares cerrados para evitar posibles acosos, lo que evidencia el impacto de la violencia de género en su trayectoria laboral.

En otros casos, algunas participantes se han visto expuestas a juicios que cosifican, cuando reciben comentarios no deseados sobre su aspecto físico en su lugar de trabajo, ante lo cual se sienten hostigadas y terminan por renunciar. Así lo relatan:

... había mucho hombre de mineras o construcciones o que eran como muy pasados para la punta [atrevido, desvergonzado) y ahí ya no, me colapsé. Era mucho estrés. Porque tampoco puedes atenderlos mal ni 
contestarles pesado y como que colapsé. Eso fue como la dificultad que tuve ahí, por eso renuncié. (ID6, 29 años, secretaria, conviviente, sin hijos).

Sí creen que te pueden hacer comentarios cómo tú eres de apariencia. Nosotras estábamos en el casino con una amiga yendo a almorzar y se nos acerca un hombre, no lo conocíamos, y dice "no se mueva así que provoca a todos" y no lo conocía (...) creen que pueden hacerte cualquier comentario porque tú eres mujer; y sí, a muchas otras diciéndole "usted, como no se quiere, no se pinta". (...) Igual lamento no haberme ido antes (ID4, 29 años, historiadora, conviviente, sin hijos)

En estos dos casos (ID6 e ID4), donde el acoso sexual era verbal, se muestra cierta resistencia inicial ante la salida laboral, pero que termina en el colapso o estrés y finalmente incide en tomar la decisión de renunciar al trabajo.

La experiencia de acoso sexual para estas participantes es una forma de violencia de género en el trabajo que conlleva cambiar de rubro, tipo de trabajo o de ocupación, por lo que existe una relación directa entre la situación de acoso sexual en el trabajo y su efecto en la trayectoria laboral. Estas tres participantes (ID4, ID6, ID8) pertenecen a la cohorte más joven (20-35 años), aunque difieren en nivel educacional y ocupación.

\section{Discusión y Conclusiones}

El género es un eje articulador de desigualdades estructurales (Storer, Casey, Carlson, Edleson \& Tolman, 2016). Por ejemplo, incide en la distribución de recursos, condiciones laborales, autonomía y nivel de autoridad que las personas tienen (Mora, 2013). El ámbito del trabajo replica el sistema de relaciones de género que muchas veces desvaloriza y discrimina a las mujeres (Calvin Pérez et al., 2013). En un país con enormes desigualdades sociales (Han, 2012; Richards, 2013), la asimetría de poder y las discriminaciones de género en el espacio laboral (Mora \& Blanco, 2018; Undurraga, 2018, 2019a; Undurraga \& Barozet, 2015) acentúan aún más las inequidades y las posibilidades de (re)producir violencia. La asimetría en las relaciones sociales favorece la violencia en el trabajo (Díaz et al., 2017).

Los resultados de esta investigación muestran variadas formas de violencia de género en el trabajo que han experimentado mujeres profesionales y no-profesionales en Chile. Esta se manifiesta a través de: (a) un trato condescendiente, (b) discriminación frente a la maternidad y (c) acoso sexual. Estos resultados muestran diferencias y similitudes en las respuestas ante la violencia de género en el trabajo según ocupación, nivel educacional, edad y experiencias subjetivas de las participantes afectadas. Esto releva el concepto de interseccionalidad (Crenshaw, 1991; Ferguson, 2013; McCall, 2005) para mostrar cómo distintas categorías sociales se relacionan y se superponen para explicar las desigualdades sociales. En Chile, investigaciones recientes han utilizado la herramienta analítica de interseccionalidad para explicar desigualdades sociales en el mercado laboral, en particular, develando prácticas de discriminación en los procesos de reclutamiento y selección de personal (Undurraga, 2018, 2019a). Así, categorías sociales se entrecruzan en un contexto determinado para acentuar privilegios o vulnerabilidades. En este estudio, mujeres jóvenes no-profesionales (intersección de género, edad y nivel educacional) estarían más expuestas ante hechos de violencia de género en el trabajo que las profesionales. Ello vuelve manifiesta una intersección de condiciones que eventualmente limita la toma de acciones, sea por la falta de poder en relación al género, la baja posición en la jerarquía dentro de la organización o el miedo a perder la fuente laboral, cuestión aún más compleja en contextos de creciente flexibilización y precarización del mercado laboral, lo que dificulta el acceso a trabajos estables y de calidad.

Respecto de los tipos de violencia, ante tratos condescendientes, las profesionales afectadas muestran una mayor disposición a defenderse y a tomar acciones respecto de estas alteraciones. Esto podría significar que más años de estudio y un título universitario generarían en las mujeres mayor agencia para confrontar la violencia de género en el trabajo. Por el contrario, grupos más vulnerables, como mujeres jóvenes noprofesionales, donde clase, género y edad se intersectan, quedarían más a la deriva y a resignarse a situaciones de malos tratos por el hecho de ser mujer. Asimismo, la discriminación frente a la maternidad y el acoso sexual fueron más frecuentes entre mujeres no-profesionales que en profesionales. Esto muestra que el género en intersección con el nivel educacional (donde muchas veces jerarquía y clase social están relacionados) incidirían en una menor posición de poder y agencia para confrontar situaciones de violencia de género en el trabajo. Así, las desigualdades de poder (de género, clase, edad, entre otras categorías sociales) incidirían en una mayor prevalencia de violencia laboral. 
Respecto a la discriminación frente a la maternidad, los relatos de las participantes son patentes: han sufrido mobbing maternal al ver sus funciones limitadas, reducidas sus interacciones laborales y sufrido hostigamiento por estar embarazadas o por utilizar la hora legal de amamantamiento. Cabe notar que la mayoría de las afectadas tomó con resignación estas malas experiencias, privilegiando el período de gestación más que un cambio laboral inminente. No obstante, luego de dichas experiencias, tomaron razón de las injusticias sufridas por el hecho de ser madre. Esto se agudiza aún más considerando el contexto nacional: en Chile, el promedio de hijos por mujer se ha reducido en el tiempo, pasando de 1,6 hijos por mujer en 2002, a 1,3 en 2017 (INE, 2018). Esto significa que posiblemente una trabajadora hará uso del pre y post natal una o dos veces a lo largo de su vida laboral. Sin embargo, las mujeres son castigadas persistentemente en diferentes ámbitos por la posibilidad de engendrar, tales como (a) en el sistema de salud, con cotizaciones más caras que para los hombres; (b) en el ámbito laboral, como la brecha salarial de género y (c) en el sistema de pensiones, con tasas diferenciadas por sexo, lo que incide en pensiones significativamente menores para las mujeres que los hombres (Comisión Asesora Presidencial sobre el Sistema de Pensiones, 2015; ComunidadMujer, 2016; Undurraga \& Becker, 2019; Undurraga, 2019b). Todo esto acentúa las inequidades de género y las desigualdades de poder.

En el contexto laboral, la maternidad sería entendida como un asunto personal y de mujeres, que iría en contra del trabajo y de los objetivos de producción de la organización. En empresas, la conciliación trabajo-familia es entendida como un asunto femenino (Gómez-Urrutia \& Jiménez-Figueroa, 2019). Para ingresar a un nuevo trabajo o ascender laboralmente, las mujeres están en condiciones dispares frente a sus colegas hombres y expuestas a discriminaciones por el hecho de ser mujer, por su asociación con la maternidad, inclusive aquellas que no quieren o no pueden ser madres (Undurraga, 2018). Esto refleja las tensiones que derivan del modelo cultural, que asigna a las mujeres el trabajo reproductivo —el trabajo doméstico y de cuidado-, mientras a los hombres, el productivo, en un creciente contexto en que las mujeres pueden ser madres y trabajadoras buena parte de sus vidas (Gómez \& Jiménez, 2018; Gonzálvez Torralbo, 2018; Segalen, 2018; Soto, 2018). Díaz et al. (2017) plantean que, a pesar de que el discurso social brinda a la maternidad un rol fundamental en la sociedad, esta y el cuidado de otros son vistos como una interferencia negativa para el desempeño laboral.

Estas dificultades se asocian a creencias sobre los altos costos de la maternidad para los empleadores. Empero, estos supuestos no están basados en evidencia. Por ejemplo, un estudio de la Oficina Regional para América Latina de la OIT, que examinó los costos laborales de hombres y mujeres en cinco países de Latinoamérica (Chile, Brasil, Argentina, Uruguay y México), demostró que "los costos monetarios directos para el/a empleador/a asociados a la protección de la maternidad y al cuidado infantil son muy reducidos, representando en los cinco países analizados menos del $2 \%$ de la remuneración bruta mensual de las mujeres" (Abramo \& Todaro, 2002, p. 27). Estas cifras muestran la discriminación contra las mujeres, cuando se supone que la contratación de mujeres tendría altos costos, muchas veces justificando diferencias salariales entre hombres y mujeres, así como desigualdades en el ingreso, desarrollo y ascenso laboral, haciendo diferencias perjudiciales en base al género. Esto da cuenta de que el género sigue siendo un eje estructural para comprender las desigualdades en Chile, en particular, en el espacio laboral.

En cuanto al acoso sexual, esta sería la experiencia más violenta que redundaría en el retiro laboral, cambiando de rubro o de ocupación. Las experiencias de acoso sexual en el trabajo significan una experiencia negativa que implica una transición "obligada" en la trayectoria laboral; obligada entre comillas, porque algunas participantes "deciden" renunciar, cambiando de trabajo o de ocupación, escapando de una situación que no han escogido ni optado. Así, la violencia de género en el trabajo se torna un punto de inflexión en la vida laboral de estas mujeres.

En Chile, el acoso sexual se relaciona con el abuso de poder de autoridades en posiciones jerárquicas, pues la mayoría de las personas denunciadas ocupan cargos superiores: gerentes, jefaturas o representantes de la empresa (Carrasco Oñate \& Vega López, 2009). Esto sostiene el argumento que la violencia se relaciona con las desigualdades de poder. La sensación de agobio y el miedo descritos por las participantes afectadas, sumado a la incredulidad de los superiores, torna insostenible la permanencia en el trabajo. Asimismo, la sensación de impotencia y la alta rotación de ciertos tipos de trabajos hacen poco factible la exigencia de protección ante estos eventos. He aquí la relevancia de las condiciones laborales para hacer frente a estas vulneraciones, seguir las regulaciones y estándares internacionales en estas materias, así como implementar directrices preventivas (ILO, 2016; Moore, 2018; Pillinger, 2017). 
Ante experiencias de violencia de género en el trabajo en las no-profesionales afectadas, se observan reacciones de resignación y resistencia. Por un lado, la resignación ante la violencia de género en el trabajo podría atribuirse a la naturalización de la dominación masculina (Bourdieu, 1998/2000), que implica en los dominados - en este caso las mujeres - el reconocimiento de la sumisión, tanto a la autoridad (masculina) como al sistema (laboral). Ya sea por miedo a la jefatura o pares varones, a vivir un mayor daño, a perder la fuente laboral o ante la paralización frente a hechos de violencia, estas mujeres se han resignado ante condiciones que ellas consideran negativas en su trayectoria. Por otro lado, cuando se habla de dominación, también hay que considerar los espacios de resistencia: resistencia, en primer lugar, al poder nombrar y contar estas experiencias como un hito relevante en su trayectoria laboral y, en segundo lugar, resistencia al tomar acciones frente a estos hechos. En su mayoría, se trata de experiencias imborrables que las participantes no quisieran volver a recorrer. Esto cobra aún mayor interés cuando se considera que en las entrevistas no se preguntó a priori sobre eventos de violencia, sino sobre momentos importantes en la vida laboral de las personas. Los hechos de violencia relatados por las participantes fueron considerados por ellas mismas como eventos fundantes en sus trayectorias laborales. Las trayectorias laborales están imbricadas con las experiencias subjetivas, momentos biográficos y condiciones socioeconómicas. Si bien en el relato de algunas entrevistadas fue posible identificar trayectorias dinámicas producto de la flexibilización del mercado laboral y la edad de las participantes, las situaciones de abuso y violencia en el trabajo fueron hitos que acentuaron la rotación e inestabilidad laboral, particularmente entre las más jóvenes (20-35 años). Mujeres entre 40 y 50 años, que esperarían cierta estabilidad y permanencia laboral, ante hechos de violencia de género en el trabajo, se ven forzadas a mostrarse a la defensiva o bien renunciar. Entre las más jóvenes (20-35 años), aunque se observa un número de transiciones laborales mayor que en las personas de más edad (61-75; 76 y más años), las experiencias de violencia en el trabajo generaron quiebres desalentadores. Estas vivencias se intersectan con la etapa del ciclo de vida en la que se encuentran, condiciones y oportunidades del mercado laboral, así como percepciones en torno a los límites y potencialidades personales. Nuevamente, aquí el concepto de interseccionalidad es iluminador para develar las distintas posiciones de poder e interacción entre categorías sociales para la (re)producción de desigualdades sociales en el mundo laboral.

Lamentablemente, la violencia de género en el trabajo es una realidad más común de lo deseada, pero poco estudiada. Esta investigación ha contribuido a mostrar los tipos de manifestaciones de la violencia de género en el trabajo y sus implicancias en la trayectoria laboral en un grupo de mujeres chilenas. Se observa que los hitos de violencia se traducen en experiencias negativas significativas, siendo el acoso sexual el tipo de violencia más fuerte para las participantes, resultando en el retiro laboral o cambio de ocupación. Se concluye que el género es un eje articulador de la desigualdad en Chile, en este caso, respecto a lo laboral. La violencia en el trabajo es típicamente una cuestión de género: la mujer es víctima de violencia por el hecho de ser mujer, referida a la posición de poder que se le adscribe culturalmente. Si bien la violencia de género no es el único tipo de violencia presente en el espacio laboral, las cifras nacionales e internacionales sobre violencia dan cuenta de que las mujeres son las principales víctimas de violencia. Así, se reconoce que las mujeres están más expuestas a recibir violencia laboral tanto a nivel internacional (Cruz \& Klinger, 2011; ONU Mujeres, 2019; Woetzel, 2015) como en Chile (Ansoleaga Moreno et al., 2016; Carrasco Oñate \& Vega López, 2009; Díaz et al., 2017). Este estudio ha mostrado que, debido a una cultura patriarcal y a aspectos organizacionales (Chang et al., 2012; Triana et al., 2015), pueden generarse agresiones en el trabajo, perjudicando ostensiblemente las trayectorias laborales de mujeres.

Esta investigación tiene ciertas limitaciones: (a) la muestra es pequeña y (b) en las entrevistas no se condujo a priori a hechos de violencia. Probablemente si se hubiese intencionado directamente sobre eventos de violencia y maltrato en el trabajo, habría aumentado considerablemente el número de recuerdos y reporte de este tipo de hechos entre las participantes.

El género y las dinámicas que se develan a partir de este estudio dan cuenta de situaciones de vulnerabilidad incrustadas en la sociedad chilena en distintas dimensiones y espacios. El laboral no es la excepción. La violencia involucra a diferentes ámbitos, siendo este un problema sistémico. Esto exige responsabilidades por parte de los distintos actores e instituciones: organizaciones, Estado, familia, trabajadores y sindicatos. En Chile, país caracterizado por altas desigualdades de poder, inequidades de género y bajas tasas de sindicalización, es crucial aumentar la concientización sobre esta problemática, fortalecer la agencia de las y los trabajadores, ejercer las regulaciones y protecciones ante la violencia en el trabajo, así como tomar medidas preventivas para evitar estos hechos. Para ello, se insta a: generar una educación con perspectiva de género a nivel prescolar, escolar, universitario y en el espacio laboral; ampliar 
y aplicar un marco regulatorio más estricto de sanciones y protección ante hechos de violencia en el trabajo; realizar más investigación sobre violencia de género en el trabajo para abordar con mayor profundidad sus alcances; y practicar la colaboración y el respeto para generar una cultura laboral libre de violencia de género, que garantice dignidad a las personas.

\section{Referencias}

Abramo, L. \& Todaro, R. (2002). Costos laborales y reproducción social: análisis comparativo de cinco países latinoamericanos. En L. Abramo \& R. Todaro (Eds.), Cuestionando un mito: costos laborales de hombres y mujeres en América Latina (pp. 11-50). Lima, Perú: Oficina Internacional del Trabajo, Oficina Regional para América Latina y el Caribe.

Acker, J. (1990). Hierarchies, jobs, bodies: A theory of gendered organisations. Gender \& Society, 4, 139-158. https://doi.org/10.1177/089124390004002002

Acker, J. (2006). Class questions: Feminist answers. Lanham, MD: Rowman \& Littlefield.

Ansoleaga, E., Gómez-Rubio, C. \& Mauro, A. (2015). Violencia laboral en América Latina: una revisión de la evidencia científica. VERTEX: Revista Argentina de Psiquiatría, 26, 444-452. Extraído de http://www.polemos.com.ar/docs/vertex/vertex124.pdf

Ansoleaga, E. \& Urra, M. (2015). Efectos del riesgo psicosocial laboral en la salud mental de funcionarios del Servicio Médico Legal de Chile. VERTEX: Revista Argentina de Psiquiatría, 26, 427-434. Extraído de http://www.polemos.com.ar/docs/vertex/vertex124.pdf

Ansoleaga Moreno, E., Díaz Berr, X. \& Mauro Cardarelli, A. (2016). Gendered work violence issues and mental health among Chilean women workers. En J. Gideon (Ed.), Handbook on gender and health (pp. 203-219). Cheltenham, Reino Unido: Edward Edgar.

Artigas, M. V. (2007). Violencia en el trabajo. FACES, 13(28), 71-85. Extraído de https://eco.mdp.edu.ar/cendocu/repositorio/FACES_n28_71-85.pdf

ATLAS.ti Scientific Software Development GmbH. (2020). https://atlasti.com

Bourdieu, P. (1998/2000). La dominación masculina.(J. Jordá Trad.; Título original: La domination masculine). Barcelona, España: Anagrama.

Calvin Pérez, M. E., Matamala Vivaldi, M. I., Eguiguren Bravo, P., Gálvez Pérez, T., Berlagoscky Mora, F., Díaz Berr, X., Iglesias Saldaña, M.; Güida Leskevicius, C. \& Hevia Rivas, P. (2013). Violencia de género en Chile. Informe monográfico $2007-2012$. Santiago, Chile: Observatorio de Equidad de Género en Salud/Organización Panamericana de la Salud. Extraído de https://www.paho.org/chi/index.php?option=com_docman\&view=download\&alias=123-violencia-de-genero-en-chile-informemonografico-2007-2012\&category_slug=sistema-de-salud\&Itemid=1145

Cárdenas Tomažič, A. (2018). Segregación laboral según género: ¿un techo de vidrio o de metal? En A. Cárdenas Tomažič \& A. M. Yévenes Ramírez (Comps.), Familia(s), mujer(es) y trabajo(s): un debate internacional (pp. 87-121). Buenos Aires, Argentina: Teseo.

Cárdenas, A., Undurraga, R., Altschwager, C., Leiva, M., Badilla, M. \& Prado, X. (2014). Informe final: estudio trayectorias laborales de la clase media-baja en Chile. Santiago, Chile: Programa de las Naciones Unidas para el Desarrollo. Extraído de http://www.cl.undp.org/content/chile/es/home/library/poverty/documentos_de_trabajo/informe-final--estudio-trayectorias-laborales-dela-clase-media-.html

Carrasco Oñate, C. \& Vega López, P. (2009). Acoso sexual en el trabajo żdenunciar o sufrir en silencio?: análisis de denuncias (Aporte al Debate Laboral N ${ }^{\circ}$ 23). Santiago, Chile: Dirección del Trabajo, División de Estudios.

Castillo, J. \& Cabezas, G. (2010). Caracterización de jóvenes primera generación en educación superior. Nuevas trayectorias hacia la equidad educativa. Calidad en la Educación, 32, 44-76. https://doi.org/10.31619/caledu.n32.151

Chang, C. -H., Eatough, E. M., Spector, P. E. \& Kessler, S. R. (2012). Violence-prevention climate, exposure to violence and aggression, and prevention behavior: A mediation model. Journal of Organizational Behavior, 33, 657-677. https://doi.org/10.1002/job.776

Chappell, D. \& Di Martino, V. (2006). Violence at work ( $3^{\mathrm{a}}$ ed.). Genève, Suiza: International Labour Office.

Chile, Instituto Nacional de Estadísticas (2018). Síntesis de resultados CENSO 2017. Santiago, Chile: Autor. Extraído de http://www.censo2017.cl/descargas/home/sintesis-de-resultados-censo2017.pdf

Chile, Instituto Nacional de Estadísticas (2019). Boletín estadístico: empleo trimestral (Edición No 251). Santiago, Chile: Autor. Extraído de https://www.ine.cl/docs/default-source//ocupacion-y-desocupacion/boletines//2019/pais/bolet\%C3\%ADn-empleo-nacional-trimestrem\%C3\%B3vil-junio-julio-agosto-2019.pdf?sfvrsn= df1808af_4

Cockburn, C. (1983). Brothers: Male dominance and technological change. London, Reino Unido: Pluto Press.

Comisión Asesora Presidencial sobre el Sistema de Pensiones (2015). Brechas de género y pensiones en Chile. Santiago, Chile: Autor. Extraído de http://www.comision-pensiones.cl

ComunidadMujer (2016). Informe GET. Género, educación y trabajo. La brecha persistente. Primer estudio sobre la desigualdad de género en el ciclo de vida. Una revisión de los últimos 25 años. Santiago, Chile: Extraído de informeget.cl/wpcontent/uploads/2016/06/Informe-GET.pdf

ComunidadMujer (2018). Informe GET 2018. Género, educación y trabajo: avances, contrastes y retos de tres generaciones. Santiago, Chile: Autor. Extraído de http://www.comunidadmujer.cl/biblioteca-publicaciones/wp-content/uploads/2018/10/INFORME-GET2018_Tres-Generaciones.pdf

Crenshaw, K. (1991). Mapping the margins: Intersectionality, identity politics, and violence against women of color. Stanford Law Review, 43, 1241-1299. https://doi.org/10.2307/1229039

Cruz, A. \& Klinger, S. (2011). Gender-based violence in the world of work: Overview and selected annotated bibliography (Working Paper 3). Genève, Suiza: International Labour Office. Extraído de https://www.ilo.org/wcmsp5/groups/public/---dgreports/--gender/documents/publication/wcms_155763.pdf

De la Lastra, C. \& Campusano, C. L. (2006). Trayectorias laborales juveniles. En M. Charlin de Groote \& J. Weller (Eds.), Juventud y mercado laboral: brechas y barreras (pp. 51-81). Santiago, Chile: Facultad Latinoamericana de Ciencias Sociales/Comisión Económica para América Latina y el Caribe. Extraído de https://repositorio.cepal.org/bitstream/handle/11362/31952/S2006356_es.pdf

Díaz Berr, X., Mauro Cardarelli, A., Ansoleaga Moreno, E. \& Toro Cifuentes, J. P. (2017). Violencia de género en el trabajo en Chile. Un campo de estudio ignorado. Ciencia \& Trabajo, 19, 42-48. https://doi.org/10.4067/S0718-24492017000100042

Dirección del Trabajo (2019). ¿Qué es el acoso laboral? Santiago, Chile: Dirección del Trabajo, Centro de Consultas. Extraído de http://www.dt.gob.cl/portal/1628/w3-article-99176.html 
Espinar Ruiz, E. \& Mateo Pérez, M. A. (2007). Violencia de género: reflexiones conceptuales, derivaciones prácticas. Papers: Revista de Sociología, 86, 189-201. https://doi.org/10.5565/rev/papers/v86n0.817

Ferguson, S. J. (Ed.) (2013). Race, gender, sexuality, \& social class: Dimensions of inequality. Thousand Oaks, CA: SAGE.

Flick, U. (1998/2004). Introducción a la investigación cualitativa. (T. del Amo, Trad.; Título original: An introduction to qualitative research), Madrid, España: Morata.

Glaser, B. G. \& Holton, J. (2004). Remodeling grounded theory. Forum: Qualitative Social Research, 5(2), art. 4. https://doi.org/10.17169/fqs-5.2.607

Glaser, B. G. \& Strauss, A. L. (1967). The discovery of grounded theory. Chicago: Aldine Press.

Goldblatt, H. \& Buchbinder, E. (2003). Challenging gender roles: The impact on female social work students of working with abused women. Journal of Social Work Education, 39, 255-275. Extraído de www.jstor.org/stable/23044064

Gómez, V. \& Jiménez, A. (2018). Equilibrio trabajo-familia, un desafío para la igualdad y el desarrollo. En C. Mora, A. Kottow, V. Osses \& M. Ceballos (Eds.), El género furtivo: la evidencia interdisciplinar del género en el Chile actual (pp. 141-157). Santiago, Chile: LOM.

Gómez-Urrutia, V. \& Jiménez-Figueroa, A. (2019). Género y trabajo: hacia una agenda nacional de equilibrio trabajo-familia en Chile. Convergencia: Revista de Ciencias Sociales, 79, artículo 7. https://doi.org/10.29101/crcs.v0i79.10911

Gonzálvez Torralbo, H. (2018). Género, cuidados y vejez: mujeres "en el medio" del trabajo remunerado y del trabajo de cuidado en Santiago de Chile. Prisma Social, 21, 194-218. Extraído de https://dialnet.unirioja.es/servlet/articulo? codigo=6521442

Guzmán, V. \& Mauro, A. (2001). Cambios generacionales en las trayectorias laborales de las mujeres. Proposiciones, 32, $190-208$. Extraído de www.sitiosur.cl/publicaciones/Revista_Proposiciones/PR-0032-3304.pdf

Guzmán, V. \& Mauro, A. (2004). Las trayectorias laborales de mujeres de tres generaciones: coacción y autonomía. En R. Todaro \& S. Yáñez (Eds.), El trabajo se transforma: relaciones de producción y relaciones de género (pp. 207-245). Santiago, Chile: Centro de Estudios de la Mujer.

Guzmán, V., Mauro, A. \& Araujo, K. (1999). Trayectorias laborales de mujeres: cambios generacionales en el mercado de trabajo. Santiago, Chile: Centro de Estudios de la Mujer.

Han, C. (2012). Life in debt: Times of care and violence in neoliberal Chile. Berkeley, CA: University of California Press.

Hernández Sampieri, R., Fernández Collado, C. \& Baptista Lucio, P. (2014). Metodología de la investigación (6 ${ }^{a}$ ed.). México DF, México: McGraw-Hill.

Hershcovis, M. S. \& Barling, J. (2010). Towards a multi-foci approach to workplace aggression: A meta-analytic review of outcomes from different perpetrators. Journal of Organizational Behavior, 31, 24-44. https://doi.org/10.1002/job.621

International Labour Organization (2016). Meeting of experts on violence against women and men in the world of work. Genève, Suiza: Autor. Extraído de http://www.ilo.org/wcmsp5/groups/public/---dgreports/---gender/documents/meetingdocument/wcms_522932.pdf

Kilpatrick, D. G. (2004). What is violence against women: Defining and measuring the problem. Journal of Interpersonal Violence, 19(11), 1209-1234. https://doi.org/10.1177/0886260504269679

Krause, M. (1995). La investigación cualitativa: un campo de posibilidades y desafíos. Revista Temas de Educación, 7, 19-39. Extraído de http://files.mytis.webnode.cl/200000020f1c75f2c42/Krause,\%20M.\%3B\%20La\%20investigación\%20cualitativa,\%20un\%20campo\%20de\%20posibilidades\%20y\%20desaf\%C3\%ADos.pdf

Krug, E. G., Dahlberg, L. L., Mercy, J. A., Zwi, A. B. \& Lozano, R. (Eds.) (2003). Informe mundial sobre la violencia y la salud (Publicación Científica y Técnica $\mathrm{N}^{\circ}$ 588). Washington, DC: Organización Mundial de la Salud.

Levy, R. \& Pavie Team (2005). Why look at life courses in an interdisciplinary perspective? En R. Levy, P. Ghisletta, J. -M. Le Goff, D. Spini \& E. Widmer (Eds.), Towards an interdisciplinary perspective on the life course (Advances in Life Course Research Volume 10, pp. 3-32). Oxford, Reino Unido: Elsevier.

Lewis, S. (1997). 'Family friendly' employment policies: A route to changing organizational culture or playing about at the margins? Gender, Work \& Organization, 4, 13-23. https://doi.org/10.1111/1468-0432.00020

Lloyd, S. \& Taluc, N. (1999). The effects of male violence on female employment. Violence Against Women, 5, 370-392. https://doi.org/10.1177/10778019922181275

Macmillan, R. (2001). Violence and the life course: The consequences of victimization for personal and social development. Annual Review of Sociology, 27, 1-22. https://doi.org/10.1146/annurev.soc.27.1.1

Madero-Cabib, I., Undurraga, R. \& Valenzuela, C. (2019). How have women's employment patterns during young adulthood changed in Chile? A cohort study. Longitudinal and Life Course Studies, 10(3), 375-397. https://doi.org/10.1332/175795919X15628474680736

Mauro, A. (2004). Trayectorias laborales en el sector financiero. Recorridos de las mujeres (Serie Mujer y Desarrollo $\mathrm{N}^{\circ} 59$ ). Santiago, Chile: Comisión Económica para América Latina y el Caribe.

Mauro, A. \& Yáñez, S. (2005). Trayectorias laborales y previsión social en Chile en un contexto de flexibilidad. Resultados de una investigación longitudinal (Cuadernos de Investigación $\mathrm{N}^{\circ}$ 1). Santiago, Chile: Centro de Estudios de la Mujer.

McCall, L. (2005). The complexity of intersectionality. Signs: Journal of Women in Culture and Society, 30, 1771-1800. https://doi.org/10.1086/426800

Moore, P. V. (2018). The threat of physical and psychosocial violence and harassment in digitalized work. Genève, Suiza: International Labour Organization. Extraído de http://www.ilo.org/wcmsp5/groups/public/---ed_dialogue/---actrav/documents/publication/wcms_617062.pdf

Mora, C. (2013). La imperceptibilidad del género. En C. Mora (Ed.), Desigualdad en Chile: la continua relevancia del género (pp. 2137). Santiago, Chile: Ediciones Universidad Alberto Hurtado.

Mora, C. \& Blanco, O. (2018). The gender deficit: Everyday practices of differentiation in the Chilean labour market. Bulletin of Latin American Research, 37, 291-305. https://doi.org/10.1111/blar.12672

Organización de las Naciones Unidas (2018). Objetivos de desarrollo sostenible. 5: Igualdad de género. New York, NY: Autor. Extraído de https://www.un.org/sustainabledevelopment/es/gender-equality/

Organización de los Estados Americanos (2018). Convención interamericana para prevenir, sancionar y erradicar la violencia contra la mujer (Convención de Belém do Pará). Washington, DC: Autor. Extraído de https://www.oas.org/es/mesecvi/docs/BelemDoPara-ESPANOL.pdf

Organización Internacional del Trabajo (2003). Repertorio de recomendaciones prácticas sobre la violencia en el lugar de trabajo en el sector de los servicios y medidas para combatirla. Genève, Suiza: Autor. Extraído de http://www.ilo.org/wcmsp5/groups/public/@ed_protect/@protrav/@safework/documents/normativeinstrument/wcms_112578.pdf

Organización Mundial de la Salud (2018). Violencia contra la mujer. Genève, Suiza: Autor. Extraído de http://www.who.int/topics/gender_based_violence/es/ 
Palma, A., Ansoleaga, E. \& Ahumada, M. (2018). Violencia laboral en trabajadores del sector salud: revisión sistemática. Revista Médica de Chile, 146, 213-222. https://doi.org/10.4067/s0034-98872018000200213

Parra Osorio, L. \& Acosta Fernández, M. (2010). La investigación cuantitativa del acoso psicológico laboral en los sectores de la educación superior y de la salud. Una revisión sistemática. Entramado, 6(1), 158-172. Extraído de http://www.redalyc.org/articulo.oa?id=265419646010

Pillinger, J. (2017). Violence and harassment against women and men in the world of work: Trade union perspectives and action. Genève, Suiza: International Labour Organization. Extraído de http://www.ilo.org/wcmsp5/groups/public/---ed_dialogue/--actrav/documents/publication/wcms_546645.pdf

Piñuel, I. (2012). Mobbing maternal: el acoso psicológico contra las mujeres embarazadas. Madrid, España: Autor. Extraído de http://acosopsicologico.blogspot.com/2012/03/mobbing-maternal-y-violencia.html?q=mobbing+maternal

Poblete Troncoso, M. \& Valenzuela Suazo, S. (2005). Enfermeras en riesgo: violencia laboral con enfoque de género. Index de Enfermería, 14(51), 40-44. https://doi.org/10.4321/S1132-12962005000300008

Programa de las Naciones Unidas para el Desarrollo (2010). Desarrollo humano en Chile. Género: los desafíos de la igualdad. Santiago, Chile: Autor.

Richards, P. (2013). Race and the Chilean miracle: Neoliberalism, democracy, and indigenous rights. Pittsburgh, PA: University of Pittsburgh Press.

Riquelme Giagnoni, V. (2011). ¿La maternidad castigada? Discriminación y malos tratos (Aporte al Debate $\left.\mathrm{N}^{\circ} 25\right)$. Santiago, Chile: Dirección del Trabajo, Departamento de Estudios. Extraído de http://www.dt.gob.cl/portal/1629/w3-article-103042.html

Segalen, M. (2018). Mujeres, trabajo, tiempo. Un punto de vista desde el caso francés. En A. Cárdenas Tomažič \& A. M. Yévenes Ramírez (Comps.), Familia(s), mujer(es) y trabajo(s): un debate internacional (pp. 21-40). Buenos Aires, Argentina: Teseo.

Sepúlveda, L. (2016). Trayectorias educativo-laborales de jóvenes estudiantes de educación técnica en Chile: ¿tiene sentido un sistema de formación para el trabajo en la educación secundaria? Páginas de Educación, 9, 189-212.

Seymour, K. (2009). Women, gendered work and gendered violence: So much more than a job. Gender, Work \& Organization, 16, 238265. https://doi.org/10.1111/j.1468-0432.2009.00437.x

Soto, D. (2018). Proyectos de vida femeninos. (In)compatibilidad entre familia y trabajo remunerado en la modernidad. En A. Cárdenas Tomažič \& A. M. Yévenes Ramírez (Comps.), Familia(s), mujer(es) y trabajo(s): un debate internacional (pp. 211-248). Buenos Aires, Argentina: Teseo.

Storer, H. L., Casey, E. A., Carlson, J., Edleson, J. L. \& Tolman, R. M. (2016). Primary prevention is? A global perspective on how organizations engaging men in preventing gender-based violence conceptualize and operationalize their work. Violence Against Women, 22, 249-268. https://doi.org/10.1177/1077801215601247

Summers, J. K., Howe, M., McElroy, J. C., Buckley, M. R., Pahng, P. \& Cortes-Mejia, S. (2018). A typology of stigma within organizations: Access and treatment effects. Journal of Organizational Behavior, 39, 853-868. https://doi.org/10.1002/job.2279

Todaro, R. \& Yáñez, S. (Eds.). (2004). El trabajo se transforma: relaciones de producción y relaciones de género. Santiago, Chile: Centro de Estudios de la Mujer.

Toro, J. P. \& Gómez-Rubio, C. (2016). Factores facilitadores de la violencia laboral: una revisión de la evidencia científica en América Latina. Ciencia \& Trabajo, 18, 110-116. https://doi.org/10.4067/S0718-24492016000200006

Triana, M. C., Jayasinghe, M. \& Pieper, J. R. (2015). Perceived workplace racial discrimination and its correlates: A meta-analysis. Journal of Organizational Behavior, 36, 491-513. https://doi.org/10.1002/job.1988

Undurraga, R. (2012). Interviewing women in Latin America: Some reflections on feminist research practice. Equality, Diversity and Inclusion, 31, 418-434. https://doi.org/10.1108/02610151211235442

Undurraga, R. (2013). Mujer y trabajo en Chile: ¿qué dicen las mujeres sobre su participación en el mercado laboral? En C. Mora (Ed.), Desigualdad en Chile: la continua relevancia del género (pp. 113-141). Santiago, Chile: Ediciones Universidad Alberto Hurtado.

Undurraga, R. (2018). "Me preguntaron: ¿quieres tener hijos pronto?". Género y selección de personal en Chile. En A. Cárdenas Tomažič \& A. M. Yévenes Ramírez (Comps.), Familia(s), mujer(es) y trabajo(s): un debate internacional (pp. 123-161). Buenos Aires, Argentina: Teseo.

Undurraga, R. (2019a). Who will get the job? Hiring practices and inequalities in the Chilean labour market. Bulletin of Latin American Research 38(5), 575-590. https://doi.org/10.1111/blar.12888

Undurraga, R. (2019b). Desigualdades en el trabajo: inequidades en la vejez. Cuadernillo $n^{o} 2$ Serie Envejecimiento. Género y Políticas Públicas: Una mirada necesaria de la vejez (pp. 47-56). Santiago: Universidad de Chile. https://uchile.cl/u160897

Undurraga, R. \& Barozet, E. (2015). Pratiques de recrutement et formes de discrimination des femmes diplômées - le cas du Chili [Prácticas de reclutamiento y formas de discriminación a mujeres profesionales - el caso de Chile]. L'Ordinaire des Amériques, 219. https://doi.org/10.4000/orda.2357

Undurraga, R. \& Becker, J. (2019). Mujeres trabajadoras jóvenes y pensiones en Chile. Psicoperspectivas, 18(3), 1-13. https://doi.org/10.5027/psicoperspectivas-vol18-issue3-fulltext-1658

Undurraga, R. \& Calvo Miranda, C. (2019, Abril). Biogramas: una herramienta multimodal feminista para el estudio del tiempo y las trayectorias laborales. Ponencia presentada en el Primer Encuentro Nacional de Métodos y Técnicas de Investigación Social, Asociación Chilena de Metodología, Medición y Evaluación, Universidad de O'Higgins, Rancagua, Chile.

Vandevelde-Rougale, A. \& Guerrero Morales, P. (2019). Emoción, discurso managerial y resistencia: el mobbing como revelador. Psicoperspectivas, 18(3), 1-12. https://doi.org/10.5027/psicoperspectivas-vol18-issue3-fulltext-1595

Virtus Partners \& ComunidadMujer (2018). Ranking mujeres en la alta dirección 2018. Santiago, Chile: Autores. Extraído de http://www.comunidadmujer.cl/biblioteca-publicaciones/wp-content/uploads/2019/01/Ranking-Mujeres-en-la-Alta-Direccio\%CC\%81n2018.pdf

Walby, S. (1990). Theorizing patriarchy. Oxford, Reino Unido: Blackwell.

West, C. \& Zimmerman, D. H. (1987). Doing gender. Gender \& Society, 1, 125-151. https://doi.org/10.1177/0891243287001002002

Fecha de recepción: Octubre de 2018.

Fecha de aceptación: Noviembre de 2019. 